\title{
Strong flows of dilute suspensions of microstructure
}

Andrew J. Szeri*

Department of Mechanical and Environmental Engineering, University of California, Santa Barbara, California 93106

Stephen Wiggins

Applied Mechanics, California Institute of Technology, Pasadena, California 91125

and Center for Nonlinear Studies, Los Alamos National Laboratory, Los Alamos, New Mexico 87545

L. Gary Leal

Department of Chemical and Nuclear Engineering, University of California, Santa Barbara, California 93106

(Received 28 August 1990)

We consider dilute suspensions that have a microstructure that may be characterized by an axial state vector. Examples include axisymmetric particles, line elements of the fluid itself, or, as an approximation, droplets of fluid or polymer molecules. Past studies, in which sufficient conditions for stretch or coherent orientation of the microstructure are obtained for steady flows with homogeneous velocity gradient tensors are shown not to apply to the general situation. Instead, a careful analysis of the microdynamical equations reveals that stretching and orientation of the microstructure by the flow must be analyzed over a time interval. Using techniques from the theory of dynamical systems, a quantitative measure is developed to determine orientations and/or stretched lengths of the microstructure, that are robust and attractive to nearby states. This leads to a strong flow criterion for unsteady flows with inhomogeneous velocity gradient tensors in which the effects of history dependence are apparent. A particular model system is treated in the case of general two-dimensional flow. The sensitivity of the results to changes in the modeling assumptions is investigated. 